Abstract

\title{
How Size Matters: Designing Diverse Fragment Libraries for Novel Drug Discovery ${ }^{\dagger}$
}

\author{
Yun Shi * and Mark von Itzstein * \\ Institute for Glycomics, Griffith University, Gold Coast Campus, Gold Coast, Queensland 4222, Australia \\ * Correspondence: y.shi@griffith.edu.au (Y.S.); m.vonitzstein@griffith.edu.au (M.v.I.) \\ + Presented at the 2nd Molecules Medicinal Chemistry Symposium (MMCS): Facing Novel Challenges in \\ Drug Discovery, Barcelona, Spain, 15-17 May 2019.
}

Published: 14 November 2019

\begin{abstract}
Fragment-based drug discovery (FBDD) has become a major strategy to derive novel lead candidates for both new and established therapeutic targets, as it promises efficient exploration of chemical space by employing fragment-sized (MW 300) compounds. One of the first challenges in implementing a FBDD approach is the design of a fragment library, and more specifically, the choice of its size and individual members. In order to construct a library that maximises the chances of discovering novel chemical matter, a large number of fragments with sufficient structural diversity are often sought. However, the exact diversity of a certain collection of fragments remains elusive, which hinders direct comparisons among different selections of fragments. Building upon structural fingerprints that are commonly utilised in cheminformatics, we herein introduced quantitative measures for the structural diversity of fragment libraries. Structures of commercially available fragments were retrieved from the ZINC database and filtered by physicochemical properties, after which they were subject to selections with library sizes ranging from 100 to 100,000 compounds. The selected libraries were evaluated and compared quantitatively, resulting in interesting sizediversity relationships. Our results suggested the existence of an optimal size for structural diversity and demonstrated that such quantitative measures can guide the design of diverse fragment libraries under various circumstances.
\end{abstract}

